\title{
DÜBLIN
}

Technological University Dublin

ARROW@TU Dublin

2004-01-01

\section{State feedback integral control by velocity-based multiple model networks}

\author{
Ruiyao Gao \\ Technological University Dublin \\ Aidan O'Dwyer \\ Technological University Dublin, aidan.odwyer@tudublin.ie
}

Follow this and additional works at: https://arrow.tudublin.ie/engscheleart

Part of the Controls and Control Theory Commons

\section{Recommended Citation}

Gao. Ruiyao and O'Dwyer, Aidan : State feedback integral control by velocity-based multiple model networks. Proceedings of the American Control Conference, pp. 2039-2044, Boston, USA. doi:10.21427/ t590-xj43

This Conference Paper is brought to you for free and open access by the School of Electrical and Electronic Engineering at ARROW@TU Dublin. It has been accepted for inclusion in Conference papers by an authorized administrator of ARROW@TU Dublin. For more information, please contact arrow.admin@tudublin.ie, aisling.coyne@tudublin.ie,gerard.connolly@tudublin.ie.

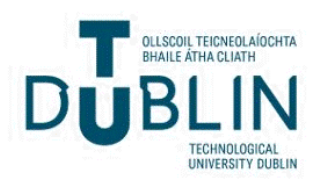




\title{
State Feedback Integral Control by Velocity-Based Multiple Model Networks
}

\author{
Ruiyao Gao, Aidan O’Dwyer, Séamus McLoone and Eugene Coyle
}

\begin{abstract}
Velocity-based (VB) multiple model networks have advantage of capturing dynamics of nonlinear systems comparing with conventional multiple model networks, for they build a direct relationship between the nonlinear system and the VB linearisation. To have the best use of modelled dynamic information for the controller design, this paper proposes a novel controller design approach, which associates an integral action with state feedback in local controller design. It overcomes the difficulty in the implementation of the velocity-based approach, which normally involves deducing the differential signal. It holds continuity with linear control theory in the analysis of the overall system and brings the potential to automatically design a controller with guaranteed performance and stability.
\end{abstract}

\section{Introduction}

In the analysis of nonlinear systems, to achieve good performance (for example, speed and accuracy) and robustness, it is desirable to derive an accurate and meaningful model of a given practical plant i.e. a model that captures the key dynamics of the plant in the operational regime of interest and provides transparent insight into the nonlinear systems. The last decade has shown an increase in the use of local model representation of nonlinear dynamics, such as gain-scheduled control ([1]), fuzzy logic systems ([2]-[3]), and local model (LM) networks ([4]), in which the locally valid sub-models are easily interpreted, and also the weighted sum of the local sub-models provides a qualitative high-level description of the nonlinear system. These approaches have the advantage of continuity with linear systems, so that well developed control methods and theory can be employed for general nonlinear systems.

However, recent research ([5]-[6]) has questioned the ease of interpretability of the multiple model frameworks for nonlinear systems and presented a novel class of blended multiple-model networks, i.e. velocity-based (VB) multiple model networks, in which the global dynamics are directly related to the local models employed. The velocity-based analysis and design framework associates a linearization with every operating point of a nonlinear system, not only the equilibrium operating points. The relationship between the nonlinear system and its VB linearisation is direct. Moreover, the underlying sub-models are continuous, velocity-based and linear, thus maintaining the continuity with existing linear techniques, which are well developed for analysis and controller design. Meanwhile, analytical results based on a complex nonlinear continuous stirred tank reactor (CSTR) process show that the velocity-based approach is ideally suited to the development of local controller (LC) networks ([7]).

Although the VB approach shows significant advantages in embodying the dynamics of the nonlinear systems, not many control applications have been developed based on VB multiple model networks, though there are some papers available ([8]-[9]). This is due to some difficulties in practical implementation that engineers have to face, for example, the differential realization of the controller input signal and the 'drift' problem of steady state errors.

This paper proposes a novel controller design approach based on the VB multiple model networks via the application of integral controllers. This approach skilfully employs the integrator to eliminate the need for differential signal of the controller input. The solution locally incorporates an integral control term in the feedback loop similar to that used in PID controllers. Meanwhile, the state observer is applied to adjust the 'drift' problem of steady state errors in the feedback loop. An illustration on a CSTR process highlights the feasibility and simplicity of the proposed approach in the application of VB multiple model networks to the control of complex nonlinear systems.

The paper is organised in the following sections. Section 2 briefly outlines the continuous-time VB multiple model network approach. Section 3 develops the state feedback integral controller based on the VB multiple model network. In section 4, the simulation results are given for a plant CSTR. Finally, the paper ends with some conclusions and suggestions for future work in section 5 .

\section{VB Multiple Model Networks}

Consider the general nonlinear state space system, with state vector $x$ and input $u$ :

$$
\begin{aligned}
\dot{x}(t) & =f(x(t), u(t)) \\
y(t) & =g(x(t), u(t))
\end{aligned}
$$

where $x \in \mathfrak{R}^{N}$, input $u \in \mathfrak{R}^{P}$. Linearizing (1) about an operating point $\left(x_{0}, u_{0}\right)$ and keeping only the linear terms yields:

$\delta \dot{x}(t)=f\left(x_{0}, u_{0}\right)+\left.\frac{\partial f}{\partial x}\right|_{\left(x_{0}, u_{0}\right)} \partial x(t)+\left.\frac{\partial f}{\partial u}\right|_{\left(x_{0}, u_{0}\right)} \partial u(t)$

Notice that if the operating point $\left(x_{0}, u_{0}\right)$ is an equilibrium point of the system, $f\left(x_{0}, u_{0}\right)=0$. This is the case for conventional local model (LM) network models, although 
there are some contributions on off-equilibrium LM networks available ([10]). However, it is not necessary to linearize the system at the equilibrium point for the VB multiple model network, which allows linearization of the system at any instant operating point.

Defining $\quad A_{0}=\left.\frac{\partial f}{\partial x}\right|_{(x, u)=\left(x_{0}, u_{0}\right)} \quad, \quad B_{0}=\left.\frac{\partial f}{\partial u}\right|_{(x, u)=\left(x_{0}, u_{0}\right)}$, equation (2) can be rewritten as

$$
\dot{x}=A_{0} x+B_{0} u+a_{0}
$$

where $a_{0}=f\left(x_{0}, u_{0}\right)-\left(A_{0} x_{0}+B_{0} u_{0}\right)$. Differentiating equation (3) with respect to time gives the linear velocity-based system

$$
\ddot{\widetilde{x}}=A_{0} \dot{\bar{x}}+B_{0} \dot{u}
$$

With the appropriate initial conditions, equations (2) and (4) give identical solutions, and therefore there is no approximation at this stage. Equation (4) establishes a direct relationship between the dynamics of the VB form of the nonlinear system and the VB linearisation near an operating point. Furthermore, members of the family of VB linearisation functions are all linear, which provides continuity with established linear theory and methods.

A velocity-based, blended, multiple-model system is formed by weighting several velocity-based linearized models as follows:

$\ddot{\tilde{x}}=\left(\sum_{i} A_{i}\left(\tilde{x}_{i}, u_{i}\right) \rho_{i}(\tilde{\psi})\right) \dot{\tilde{x}}+\left(\sum_{i} B_{i}\left(\tilde{x}_{i}, u_{i}\right) \rho_{i}(\tilde{\psi})\right) \dot{u}$

where

$A_{i}\left(\tilde{x}_{i}, u_{i}\right)=\partial f /\left.\partial \tilde{x}\right|_{(\tilde{x}, u)=\left(\tilde{x}_{i}, u_{i}\right)}, B_{i}\left(\tilde{x}_{i}, u_{i}\right)=\partial f /\left.\partial u\right|_{(\tilde{x}, u)=\left(\tilde{x}_{i}, u_{i}\right)}$ and $\left(\tilde{x}_{i}, u_{i}\right)$ is the freezing point of the $i^{t h}$ local model:

$$
\ddot{\bar{x}}=A_{i}\left(\tilde{x}_{i}, u_{i}\right) \dot{\bar{x}}+B_{i}\left(\tilde{x}_{i}, u_{i}\right) \dot{u}
$$

in which $\bar{x}$ is the state vector of the linearization function at $\left(\tilde{x}_{i}, u_{i}\right)$. The normalised weighting function is given by $\rho_{i}(\tilde{\psi})$, which is most often taken as Gaussian functions and $\tilde{\psi}$ is the scheduling vector defined by states, system input and/or output. Now we consider the dynamics of the blended system at the operating point $\left(\tilde{x}_{0}, u_{0}\right)$. The velocity-based linearized form of (5) at $\left(\tilde{x}_{0}, u_{0}\right)$, is simply obtained by freezing the validity function $\rho_{i}(\tilde{\psi})$ at the operating point and leads to the following linear system:

$$
\ddot{\tilde{x}}=\left(\sum_{i} A_{i}\left(\tilde{x}_{i}, u_{i}\right) \rho_{i}\left(\tilde{\psi}_{0}\right)\right) \dot{\tilde{x}}+\left(\sum_{i} B_{i}\left(\tilde{x}_{i}, u_{i}\right) \rho_{i}\left(\tilde{\psi}_{0}\right)\right) \dot{u}(7)
$$

With the appropriate initial conditions, the solution to (7) is initially tangential to the solution of the velocity-based multiple model system in (5). The dynamics of the multiple model system local to an arbitrary operating point are therefore the same as the dynamics of the corresponding frozen-form linear system at the same operating point. Rewriting (7) as

$$
\ddot{\tilde{x}}=\sum_{i} \rho_{i}\left(\tilde{\Psi}_{0}\right)\left(A_{i}\left(\tilde{x}_{i}, u_{i}\right) \dot{\tilde{x}}+B_{i}\left(\tilde{x}_{i}, u_{i}\right) \dot{u}\right)
$$

Equation (8) clearly highlights this direct relationship between the frozen-form (7) of the velocity-based blended system and the underlying local models (8) at $\left(\tilde{x}_{0}, u_{0}\right)$. Thus, at any arbitrary operating point, the global dynamics of the multiple model system are described by a straight-forward weighted sum of the local model dynamics. Further detailed theoretical analysis of the velocity-based nonlinear representations can be found in ([6], [7]).

\section{State Feedback and Integral Control}

Originally, integral action was employed in controller design to overcome the problem of steady state errors. In many cases, it is difficult to obtain an accurate value for the plant gain, in part, because plants are typically nonlinear and the plant model is linearized at a particular point. Therefore, steady state errors will result even though the model is sufficiently accurate for good feedback controller design. The solution is to include an integral term in the controller design.

The objective of introducing integral action here has another advantage, which is that it simplifies the implementation of the controller design in practice, based on VB multiple model networks. No numerical differential is needed for implementation of VB linearized feedback controllers, so no approximation is needed at this stage. Therefore, the controller design has the best use of dynamic information available from the VB multiple model networks, which represent the entire dynamics of the nonlinear system.

For each VB local model, a state space integral controller can be designed with the purpose of achieving satisfactory dynamic response in terms of rise-time, overshoot, settling time or other measures of transient response. A general structure of an integral controller is shown in Figure 1.

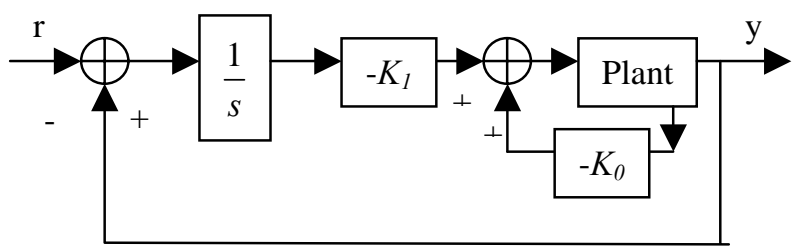

Fig. 1 Conventional state feedback integral controller

Given a linear system as

$$
\begin{aligned}
& \dot{x}=F x+G u \\
& y=H x
\end{aligned}
$$

Define $\dot{x}_{I}=y-r$, and then extend equation (9) as follows: 


$$
\left[\begin{array}{c}
\dot{x}_{I} \\
\dot{x}
\end{array}\right]=\left[\begin{array}{cc}
0 & H \\
0 & F
\end{array}\right]\left[\begin{array}{c}
x_{I} \\
x
\end{array}\right]+\left[\begin{array}{l}
0 \\
G
\end{array}\right] u-\left[\begin{array}{l}
1 \\
0
\end{array}\right] r
$$

Define $\mathrm{u}=-\left[K_{l} K_{0}\right]\left[\begin{array}{c}x_{I} \\ x\end{array}\right]$ and substitute it into (10), gives

$$
\left[\begin{array}{c}
\dot{x}_{I} \\
\dot{x}
\end{array}\right]=\left[\begin{array}{cc}
0 & H \\
-G K_{1} & F-G K_{0}
\end{array}\right]\left[\begin{array}{c}
x_{I} \\
x
\end{array}\right]-\left[\begin{array}{l}
1 \\
0
\end{array}\right] r
$$

We set the eigenvalues of the extended matrix $\left[\begin{array}{cc}0 & H \\ -G K_{1} & F-G K_{0}\end{array}\right]$ to the desired poles, and then $K_{l}$ and $K_{0}$ can be solved.

Recall the velocity-based linearization local model (6), for the $i^{\text {th }}$ VB local model, we rewrite the equation (6) as follows with definition $w=\dot{\bar{x}}$,

$$
\dot{w}=A_{i} w+B_{i} \dot{u}
$$

Let $\dot{u}=-\left[\begin{array}{ll}K_{1 i} & K_{0 i}\end{array}\right]\left[\begin{array}{c}w_{I} \\ w\end{array}\right]$, in which $\dot{w}_{I}=\dot{y}_{p}-\dot{r}$. An integral controller can be designed to satisfy some specifically assigned requirement using the above method. Then a little change can be made from Figure 1 to Figure 2, in which the system input signal is a step signal rather than the differential of it i.e. an impulse input signal.

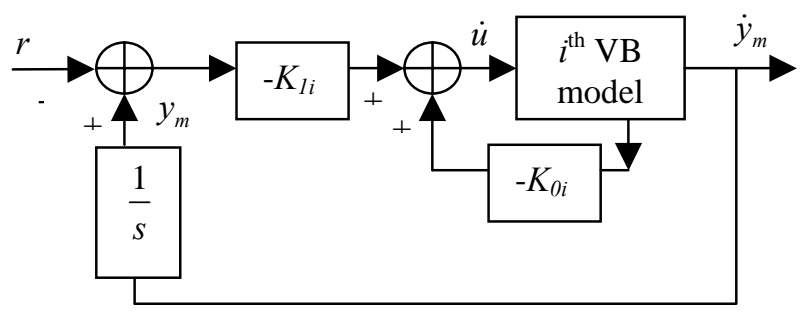

Fig. 2 The $i^{t h}$ local velocity-based integral controller

As for the blending VB multiple model networks, under the definition of $w=\dot{\tilde{x}}$, equation (7) is rewritten as

$$
\dot{w}=\sum_{i} \rho_{i} A_{i} w+\sum_{i} \rho_{i} B_{i} \dot{u}
$$

It should be emphasized that the VB multiple model networks do not have their own local states; instead, they share a 'common' state defined by the controller state vector $\dot{u}(t)$ as determined from (12). The controlled closed-loop system is described by

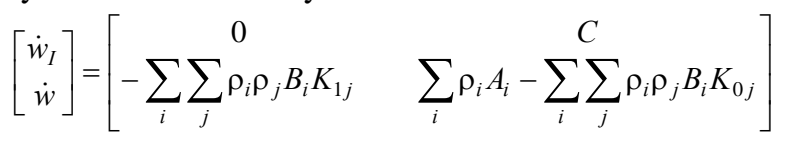

$$
\cdot\left[\begin{array}{c}
w_{I} \\
w
\end{array}\right]-\left[\begin{array}{l}
1 \\
0
\end{array}\right] \dot{r}
$$

Then the characteristic matrix of (14)

$$
\left[\begin{array}{cc}
0 & C \\
-\sum_{i} \sum_{j} \rho_{i} \rho_{j} B_{i} K_{1 j} & \sum_{i} \rho_{i} A_{i}-\sum_{i} \sum_{j} \rho_{i} \rho_{j} B_{i} K_{0 j}
\end{array}\right]
$$

can be written as

$$
\begin{aligned}
& \sum_{i} \sum_{j} \rho_{i} \rho_{j}\left[\begin{array}{cc}
0 & C \\
-B_{i} K_{1 j} & A_{i}-B_{i} K_{0 j}
\end{array}\right] \\
& =\sum_{i} \rho_{i}^{2}\left[\begin{array}{cc}
0 & C \\
-B_{i} K_{1 i} & A_{i}-B_{i} K_{0 i}
\end{array}\right]+ \\
& 2 \sum_{i<j}\left[\begin{array}{cc}
\frac{-\left(B_{i} K_{1 j}+B_{j} K_{1 i}\right)}{2} & \frac{\left(A_{i}-B_{i} K_{0 j}\right)^{C}+\left(A_{j}-B_{j} K_{0 i}\right)}{2}
\end{array}\right]
\end{aligned}
$$

Employing the stability results discussed in ([3]), the stability of system (14) can be investigated. If there is a common definite positive matrix $P$ existing for each local feedback system, the overall closed-loop system is stable and it stabilizes to the origin. This shows another advantage of the VB approach over the conventional affine LM network, which is a bounded system generally ([11]). The overall closed-loop system is shown in Figure 3.

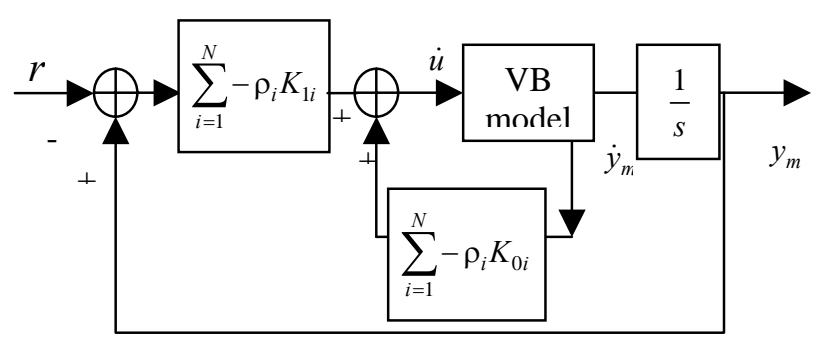

Fig. 3 Velocity-based controller network

When the VB integral controller network is applied to the plant, we propose a framework as in Figure 4, which introduces an observer to the modelling loop. The velocitybased multiple model networks have a weakness in static modelling accuracy, for there are steady state errors existing ([7]), although the VB approach shows an attractive capability in capturing the dynamics of nonlinear systems. Moreover, these steady state errors accumulate as the simulation continues and the VB multiple model outputs drift away from the nonlinear system output. So, it is desirable to bring the VB multiple model outputs back to the proper operating point when we design a model-based controller as shown in Figure 4. 


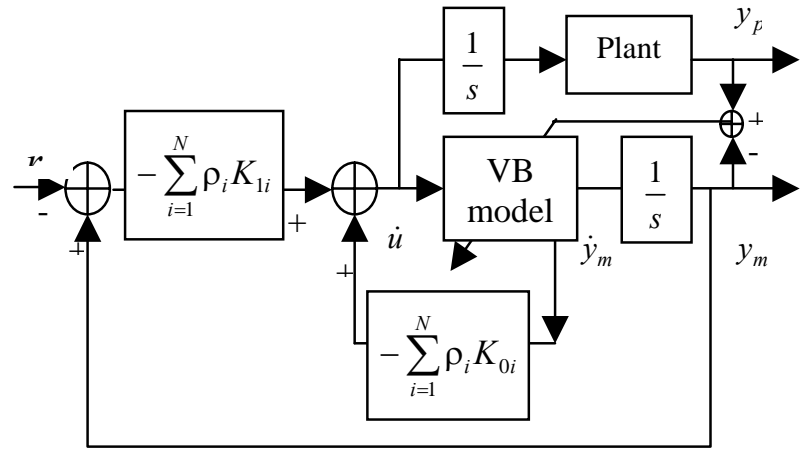

Fig. 4 VB controller network application to a plant

\section{Illustration}

\section{A. CSTR Plant}

In simulation, the continuous stirred tank reactor (CSTR) is considered in a case study. The process model consists of two non-linear ordinary differential equations ([12]),

$$
\begin{aligned}
\dot{T}(t)= & \frac{q_{f}}{V}\left(T_{f}-T(t)\right)+K_{1} C(t) \exp \left(-\frac{E}{R T(t)}\right) \\
& +K_{2} q_{c}(t)\left[1-\exp \left(-\frac{K_{3}}{q_{c}(t)}\right)\right]\left(T_{c f}-T(t)\right) \\
\dot{C}(t)= & \frac{q_{f}}{V}\left(C_{f}-C(t)\right)-K_{0} C(t) \exp \left(-\frac{E}{R T(t)}\right)
\end{aligned}
$$

It is a single input, single output process, where the input is the flow rate of a coolant $q_{c}(t)$ and the output is the concentration of a product compound $C(t)$. The reaction is exothermic which changes temperature output $T(t)$ by adjusting the effluent flow rate $q_{c}(t)$. The induction of a coolant allows manipulation of the temperature and, hence, control of the concentration. The model parameters defined, and the nominal operating conditions are shown in table 1 . The objective of the controller design is to control the concentration output $C(t)$.

Table 1. Nominal CSTR operating conditions

\begin{tabular}{|l|c|}
\hline$q_{f}=100 \mathrm{l} / \mathrm{min}$, product flow rate & $K_{2}=0.01 / \mathrm{l}$, constant \\
\hline$C_{f}=1 \mathrm{~mol} / \mathrm{l}$ input concentration & $\mathrm{K} 3=700 \mathrm{l} / \mathrm{min}$. constant \\
\hline$T_{c f}=350 \mathrm{~K}$, temperature of coolant & $T_{f}=350 \mathrm{~K}$, input temprature \\
\hline$E / R=104 \mathrm{~K}$, activation energy & $\mathrm{K} 1=1.44 * 1013 \mathrm{Kl} / \mathrm{min} / \mathrm{mol}$, \\
\hline$K_{0}=7.2 * 10^{10}$ min- 1 , constant & $V=100 \mathrm{l}$, container volume \\
\hline
\end{tabular}

The CSTR plant is highly nonlinear with exponential terms and product terms. Furthermore, open-loop step tests show that the output concentration responses vary from overdamped to under-damped, indicating the variable dynamics in the CSTR process. Figure 5 is the step response of concentration output $C(t)$ when the coolant flow rate $q_{c}(t)$ varies from $85 \mathrm{l} / \mathrm{min}$ to $111 \mathrm{l} / \mathrm{min}$.

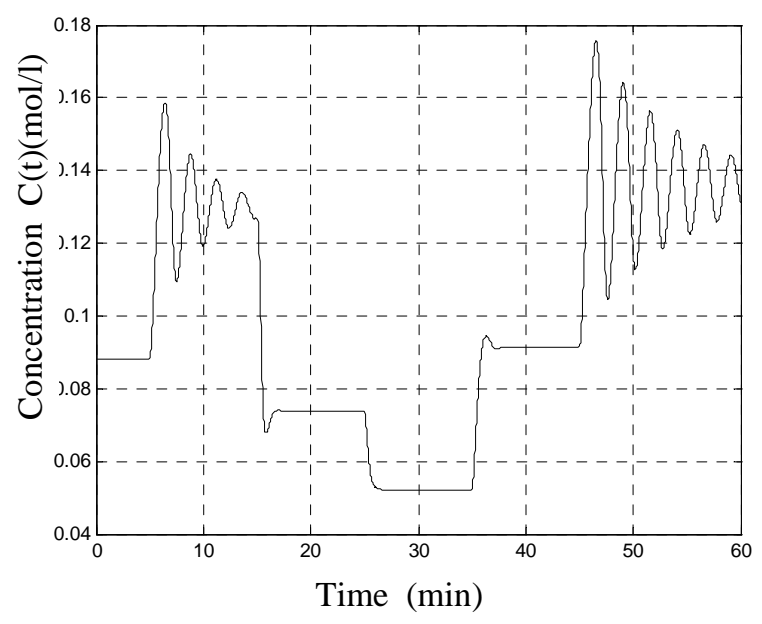

Fig. 5 Dynamic response of the CSTR plant

\section{B. Simulation Results}

The model used here consists of two nominated velocitybased local models as shown in ([7]), each corresponding to a certain range of the operating regime. These two local models are obtained by freezing the nonlinear velocity model at the appropriate linearisation points:

$C_{o}^{1}=0.062 \mathrm{~mol} / \mathrm{l}, T_{o}^{1}=448.7522 \mathrm{~K}, q_{c o}^{1}=90.0 \mathrm{l} / \mathrm{min}$

$C_{o}^{2}=0.1298 \mathrm{~mol} / \mathrm{l}, T_{o}^{1}=432.9487 \mathrm{~K}, q_{c o}^{1}=110.0 \mathrm{l} / \mathrm{min}$

in which $\left(C_{o}^{i}, T_{o}^{i}, q_{c o}^{i}\right)$ denotes the linearisation point of the $i^{\text {th }}$ local model. The integral controller design is carried out based on the controller structure outlined in section 3 . The first local controller corresponding to the velocity-based local model at $\left(C_{o}^{1}, T_{o}^{1}, q_{c o}^{1}\right)$ is designed to give a dominant set of closed-loop poles with a settling time of approximately 0.5 minutes, and the second local controller corresponding to the velocity-based local model at $\left(C_{o}^{2}, T_{o}^{2}, q_{c o}^{21}\right)$ is designed to give a dominant set of closed-loop poles with a settling time of approximately 0.25 minutes. The corresponding gains designed are as follows:

$K_{11}=26285 ; K_{12}=25344 ;$

$K_{01}=\left[\begin{array}{ll}-17.4651 & -62.5806\end{array}\right]$;

$K_{02}=\left[\begin{array}{ll}-416.2 & 18807\end{array}\right]$.

$K_{j i}$ denotes the $j^{\text {th }}$ gain for the $i^{\text {th }}$ local model. Two sets of step changes are designed in the relatively over-damped operating regime $(C(t)<0.1)$ and in the relatively underdamped operating regime $(C(t)>0.1)$. The simulation results are shown in Figure 6. 


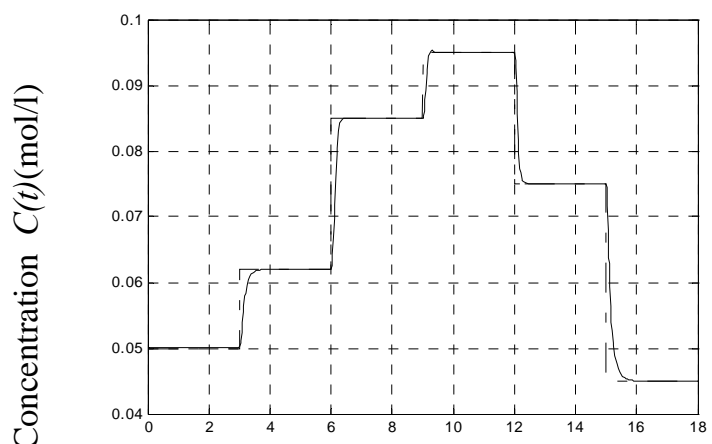

Time (min)

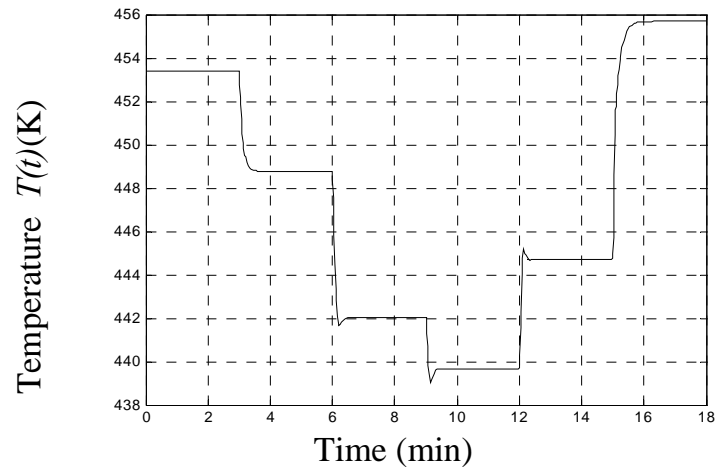

(a) Over-damped operating regimes

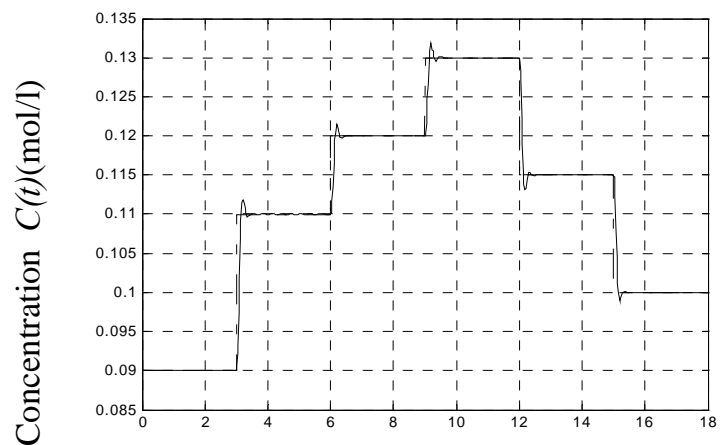

Time (min)

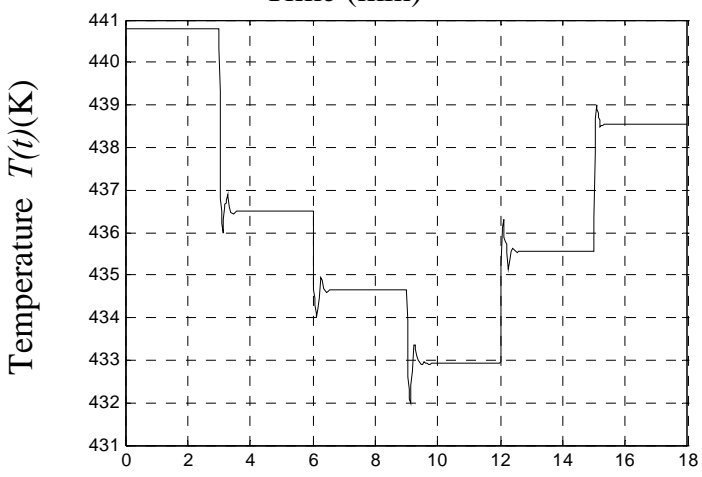

Time (min)

(b) Under-damped operating regimes

Fig. 6 Closed-loop step response
The tracking performance of the concentration output and the temperature output is reasonably good for both over-damped and under-damped operating regimes. Both the concentration outputs and temperature outputs are able to follow the step changes in less than 0.5 minutes. As expected in the design specification, in Figure 6 (a), the response generally takes longer to follow the step changes than in Figure 6 (b). Although the response in Figure 6 (b) shows a little overshoot, it settles down quicker than in Figure 6 (a) generally.

In addition, the regulation performance of the proposed integral controlled system based on VB multiple model networks is examined by adding pulse disturbances to the system. The simulation result is shown in Figure 7.
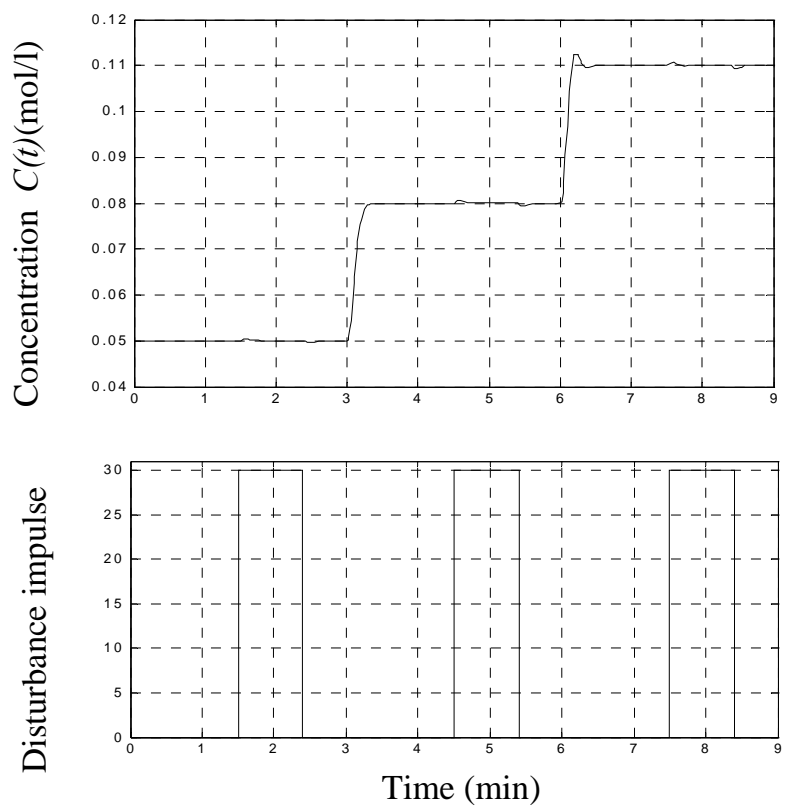

Fig. 7 Regulation test of closed-loop system

It is worthwhile to notice that only the change in the disturbance affects the concentration output. The concentration output goes back to the set point quickly after a tiny vibration, which is very limited, when the disturbance change occurs.

Another simulation example is shown in Figure 8, which compares the regulation performance of the proposed integral controlled system based on the VB multiple model networks with the gain-scheduled local controller (LC) network proposed in ([13]). Obviously, the integral controller based on VB multiple model networks allows much better robustness performance compared with the gain-scheduled local controller networks. As in figure 7, only the up/down edge of the impulse affects the concentration output, whose change is of very low amplitude. This result also, in part, demonstrates the advantage of the velocity-based linearization, which is not only applied at the equilibrium point but at any operating point, in capturing the dynamics 
instantly, so that the instant change of system dynamics can be fed back to the closed-loop and be properly controlled.
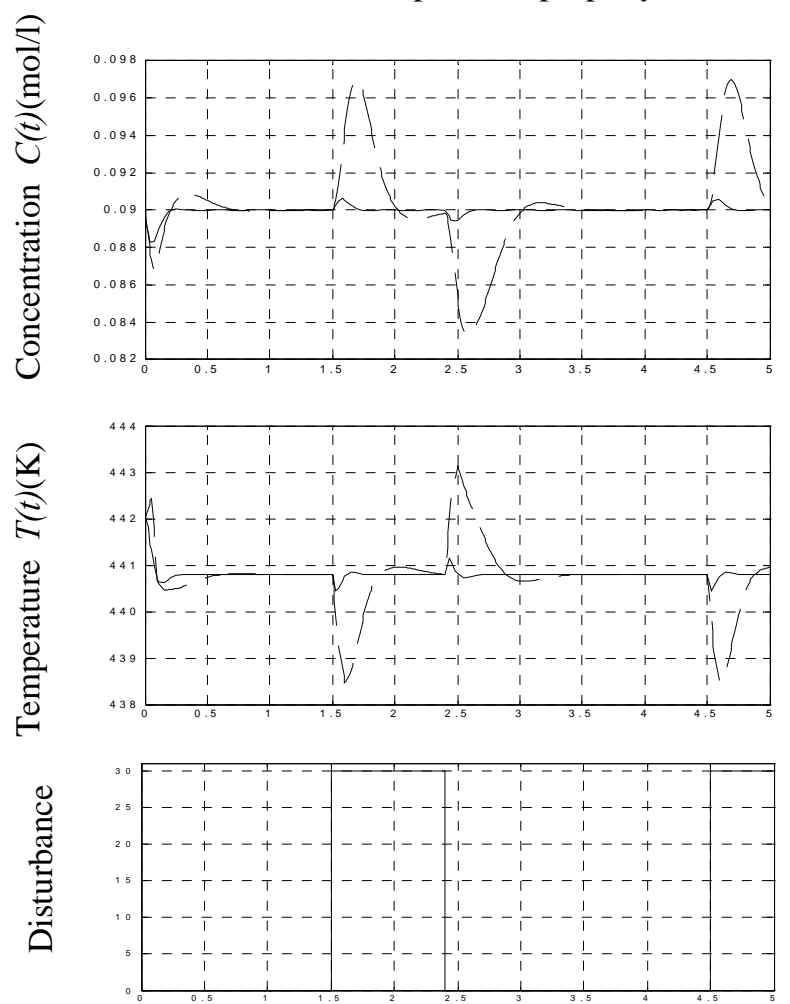

Time (min)

Fig. 8 Comparison of regulation performance

The solid line is from the integral controlled system based on VB multiple model networks and the dashed line is from the gain-scheduled LC network controlled system.

\section{Conclusions}

State feedback controller design has been widely applied in control. The novel element of the proposed approach is that it introduces a combination of an integral action and the state feedback control, for the controller design based on the VB multiple model networks. It skilfully utilizes the integral action to compensate for the weakness of differential action, which is normally not feasible in practical implementation but is required for the velocity-based approach. This approach shows simplicity in controller design and continuity with the well-established linear control methods and theories for controller design and analysis.

Moreover, the proposed integral controller design approach based on VB multiple model networks shows excellent trajectory tracking performance and strong robustness in application. This promising advantage is convincing when evaluating the potential of the application of VB multiple model networks in the control of nonlinear systems.

\section{Reference}

[1] Shamma, J.S., Athans, M., Analysis of gain- scheduled control for non-linear plants. IEEE Transactions on Automatic Control, vol.35, pp.898-907, 1990.

[2] Tagaka,T. and Sugeno,M., Fuzzy identification of systems and its applications for modelling and control. IEEE transactions on Systems, Man and Cyberbetics, Vol.15, pp.116-132, 1985.

[3] Wang, H.O., Tanaka K., Michael F. and Griffin F., An approach to fuzzy control of nonlinear systems: Stability and design issues. IEEE Transaction on Fuzzy Systems, Vol.4, No.1, pp.14-23, 1996.

[4] Johansen, T.A and Foss B.A., Constructing NARMAX models using ARMAX models. International Journal of Control, Vol.58, pp.1125-1153, 1993.

[5] Leith D.J. and Leithead W.E., Towards a theory of local model networks \& blended multiple model systems. UKACC International Conference on Control, pp.509514, 1998.

[6] Leith,D.J., and Leithead,W.E., Analytic framework blended multiple model systems using linear local models. International Journal of Control, 72, (7/8), pp.179-184, 1999.

[7] McLoone,S.C., Irwin,G.W., On nonlinear modelling using velocity-based multiple model networks. American Control Conference (ACC’01), Arlington, June, pp.25-27, 2001.

[8] Leith, D.J. Leithead, W.E., Gain-scheduled control of a skid-to-turn missile: relaxing slow variation requirements by velocity-based design. Proceedings of American Control Conference, pp.500-505, 2001.

[9] Rong Q., Irwin G.W. and McLoone S., Velovity-based local PID controller network. Proceedings of Irish Signal and Systems Conference, pp.70-76, 2001.

[10] Johansen T.A., Hunt K.J., P.J. Gawthrop and Fritz H., Off-equilibrium linearization and design of gain scheduled control with application to vehicle speed control. Control Engineering Practice, Vol.6, No.2, pp.167-180, 1998.

[11] Gao R., O’Dwyer A., McLoone S. and Coyle E., On the stability of affine blending systems, Irish Signals and Systems Conference, pp.488-493, 2003.

[12] Henson M.A and Seborg D.E., Input-Output linearisation of general non-linear processes, AIChE Journal Vol.36, 1753-1757, 1990.

[13] Gao R., O’Dwyer A. and Coyle E., Model predictive control of CSTR based on local model networks. Proceedings of Irish Signals and Systems Conference, pp.397-402, 2002. 